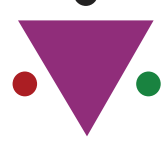

IJCRR

Section: Healthcare

Sci. Journal Impact

Factor: 6.1 (2018)

ICV: 90.90 (2018)

Scopus'

\title{
Intrathecal Nalbuphine an Addition to Anaesthesia Armamentarium to Prolong Postoperative Analgesia
}

\section{Pratibha Deshmukh ${ }^{1}$, Nikita Dhumne ${ }^{2}$, Medha Sangawar ${ }^{3}$, Vivek Chakole ${ }^{4}$}

\begin{abstract}
Professor and HOD, Department of Anaesthesiogy, Datta Meghe Medical College, Wanadongri, Hingna Road, Nagpur, Maharashtra- 441110;

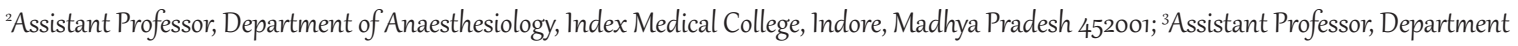
of Anaesthesiogy, Indira Gandhi Government Medical College, and Hospital, Nagpur, Maharashtra- 4400018; 4Professor, Department of Anesthesiology Jawaharlal Nehru Medical College, Datta Meghe Institute of Medical Sciences, Sawangi, Meghe, Wardha-442001.
\end{abstract}

\section{ABSTRACT}

Background: Nalbuphine is a synthetic opioid. It has mixed agonist-antagonist action. When used as an adjuvant to intrathecal bupivacaine it acts on kappa receptors in the dorsal horn of the spinal cord producing analgesia. We aimed the present study to evaluate the effectiveness of intrathecal nalbuphine as an adjuvant. At the same time, the efficacy of nalbuphine for sensorimotor characteristics and postoperative analgesia was also judged.

Method: A total of 100 patients were enrolled. They were randomly allocated into two equal groups. Group B: received 15 mg hyperbaric bupivacaine $(3 \mathrm{ml}) 0.5 \%+0.5 \mathrm{ml}$ normal saline and Group BN: received $15 \mathrm{mg}$ hyperbaric bupivacaine $(3 \mathrm{ml}) 0.5 \%+$ Inj. Nalbuphine $500 \mu \mathrm{g}(0.5 \mathrm{ml})$. The sensorimotor characteristics, duration of effective analgesia, any hemodynamic alterations, and side effects were noted.

Results: The sensory and motor block characteristics were significantly faster in group BN than group B. The duration of sensory and motor block, as well as duration of effective analgesia, was significantly prolonged in group BN in comparison with group B. Quality of analgesia during the procedure was excellent in $90 \%$ and $94 \%$ in group B and BN, respectively. Sedation score, hemodynamic parameters, and respiratory rate changes were comparable between both groups. In group B, 4\% patient complained of nausea/vomiting, intraoperative hypotension noted in $4 \%$ and post-dural puncture headache was recorded in 1 patient while in group BN bradycardia was observed in 3 patients.

Conclusion: Addition of $500 \mu \mathrm{g}$ of nalbuphine as an adjuvant to hyperbaric bupivacaine $0.5 \%$ for spinal anaesthesia provides excellent analgesia with a longer duration of effective analgesia in comparison to intrathecal hyperbaric bupivacaine $0.5 \%$ alone.

Key Words: Nalbuphine, Opioid, Analgesia, Bupivacaine, Sensorimotor, Hypotension, Bradycardia

\section{INTRODUCTION}

Spinal anesthesia is the most popular regional anesthesia technique ${ }^{1 .}$ The technique is simple to perform. The onset of action is rapid. Also, it gives profound muscle relaxation ${ }^{2,3,4}$. The major limitation is the fixed duration of action of the local anaesthetic used. Therefore, the adjuvants such as opioids, alpha 2 agonists, neostigmine, magnesium, etc. came into picture 5 .

The intrathecal opioids as an adjuvant to local anaesthetic have been found to augment intra-operative and post-operative quality of analgesia ${ }^{6}$. They act synergistically with local anesthetics and intensify the sensory block without having any effect on the sympathetic blockage. It's a routine practice that opioids are added to local anaesthetic for enhancing their effects, decreasing their dosage, and therefore decreasing the potential side effects and complications. The prolongation of the duration of postoperative analgesia is an advantage $^{7,8,9}$. The major drawback of the addition of opioids is their side effects. Out of which, respiratory depression can be serious and pruritus can be bothersome.

One of the best ways to control the intrathecal Opioid related side effects is the use of mixed agonist-antagonist opioids. Nalbuphine is one of the most recently used additives to spinal bupivacaine, considering its $\mu$ antagonist and $\kappa$ agonist mechanisms of action ${ }^{1}$. As adjunct to intrathecal local anesthetics nalbuphinecan provide good intra- and post-operative analgesia. It can also decrease the incidence and obtund the severity of $\mu$ receptor side effects ${ }^{9}$. The present study was undertaken to evaluate the efficacy of intrathecal nalbuphine

\section{Corresponding Author:}

Dr. Pratibha Deshmukh, Professor and HOD, Department of Anaesthesiogy, Datta Meghe Medical College, Wanadongri, Hingna Road, Nagpur, Maharashtra- 441110,Email: pratibhauday@gmail.com

ISSN: 2231-2196 (Print)

Received: 24.05 .2020
ISSN: 0975-5241 (Online)

Revised: 17.06 .2020
Accepted: 29.06 .2020
Published: 21.07 .2020 
as a spinal adjuvant to hyperbaric bupivacaine $0.5 \%$ in comparison with bupivacaine $0.5 \%$ alone.

\section{MATERIALS AND METHODS}

The present prospective observational study was undertaken from December 2014 to November 2016 (2years) in the Department of Anaesthesiology at a Tertiary Care Centre in Maharashtra, India. Total 100 adult patients of American Society of Anaesthesiologists ASA grade I and II, age between $18-60$ years, height $150-170 \mathrm{~cm}$, either sex undergoing elective lower abdominal or lower limb surgeries under regional anaesthesia were included in the study. We divided them into two equal groups (Group B-control group and group BN- nalbuphine group). Institutional Ethical Committee approval and written informed consent was taken from all.Patients with height $<150 \mathrm{~cm}$ or $>170 \mathrm{~cm}$, physical ASA status greater than III, contraindication to spinal anaesthesia, history of hypersensitivity to local anaesthetics or opioids, spinal deformities, patients with cardiovascular, respiratory, neurological, renal or endocrine diseases or psychiatric illness, hypovolemia, severe anaemia, pregnancy, and patients not willing for the procedure were excluded from the study.

After a detailed history and clinical examination, all relevant investigation was done for every patient with note down of demographic data. All patients were kept starving for an overnight period prior to surgery as per routine.

In the preoperative room, pulse rate, BP, respiratory rate, $\mathrm{SpO} 2$ were noted. On the table, the intravenous cannula of $18 / 20 \mathrm{G}$ was secured, and the patient was preloaded with $10 \mathrm{ml} / \mathrm{kg}$ of Ringer lactate solution over 10-30mins. A multipara monitor was used to monitor vital parameters. A baseline record of NIBP, SpO2, ECG, RR was made. None of the patients were administered sedatives in premedication. Under all aseptic precautions, in lateral decubitus position, L3- L4 interspace was identified. Quincke's $25 \mathrm{G}$ needle was inserted via the midline approach till the free flow of CSF was obtained. After positive aspiration of CSF fluid, drug mixture was injected slowly (approx. $30 \mathrm{secs}$ ) as per group allotment as: - Group B: $15 \mathrm{mg}$ hyperbaric Bupivacaine $0.5 \%(3 \mathrm{ml})+0.5 \mathrm{ml}$ normal saline and Group BN: $15 \mathrm{mg}$ hyperbaric Bupivacaine $0.5 \%(3 \mathrm{ml})+$ Inj. Nalbuphine $500 \mu \mathrm{g}(0.5 \mathrm{ml})$. Inj. Nalbuphine $500 \mu \mathrm{g}$ was measured in an insulin syringe and its volume made up to $0.5 \mathrm{ml}$ with N.S before adding to Bupivacaine which itself was drawn in a $5 \mathrm{ml}$ syringe. The total volume injected was $3.5 \mathrm{ml}$ in both the groups. Nalbuphine used in the study was preservative-free.

The time of drug injection was noted and recorded as 0 . After injection patient was turned supine slowly, sensory and motor characteristics, duration of surgery, the total duration of effective analgesia assessed on VAS score, quality of analgesia, sedation score, and vital parameters were monitored and noted. Intra and postoperative side effects were noted and treated accordingly. All patients were observed in postanaesthesia recovery area of operation theatre till the administration of rescue analgesia which was the end-point of the main study. Patients were visited at 12 and 24 hours to note about side effects and complications if any.

\section{Statistical Analysis}

Mean, standard deviation, and range were used to express the results. Frequencies were denoted number and percentage. One-way analysis of variance (ANOVA) used for multiple group comparison with POST HOC multiple comparisons between groups with LSD correction and categorical data analyzed by chi-square test. $P$ value of $<0.05$ was taken as statistically significant and P-value $<0.001$ as statistically highly significant. The statistical analysis was done using Statistical Package for Social Science evaluation (SPSS) version 20.0.

\section{OBSERVATIONS AND RESULTS}

The demographic profiles of the patients and type of procedure were comparable and found no difference between two groups to be considered as significant (Figure 1). Male preponderance was observed in both the groups. The mean duration of surgery was found to be less in group B (67.62 \pm 20.02 ; ranged from 45-105 minutes) as compared to groups $\mathrm{BN}$ (88.12 \pm 18.03 ; ranged $45-140$ minute), $(\mathrm{p}=0.0001)$.

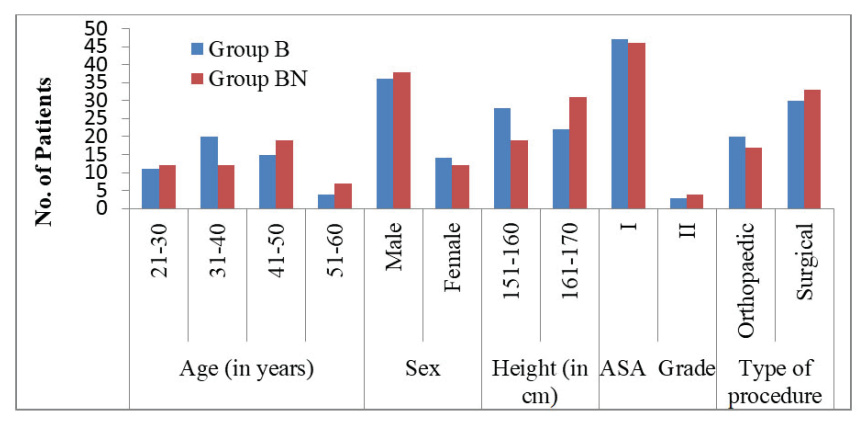

Figure 1: Comparison of demographic data and type of procedure between two groups.

The onset of sensory and motor block was significantly faster and prolonged in group BN as compared to group B. The difference between the two groups was statistically significant $(\mathrm{P}=0.0001)$. The time duration for effective analgesia in group B was significantly shorter $(234.57 \pm 41.34 \mathrm{~min})$ in comparison with group BN (371.22 $\pm 40.24 \mathrm{~min})$ as depicted in Figure 2.

The quality of analgesia during the procedure was excellent at $91 \%$ and $95 \%$ in group B and BN, respectively. Quality of analgesia was reported as good in $9 \%$ of patients in group B and $5 \%$ in group $\mathrm{BN}$. 


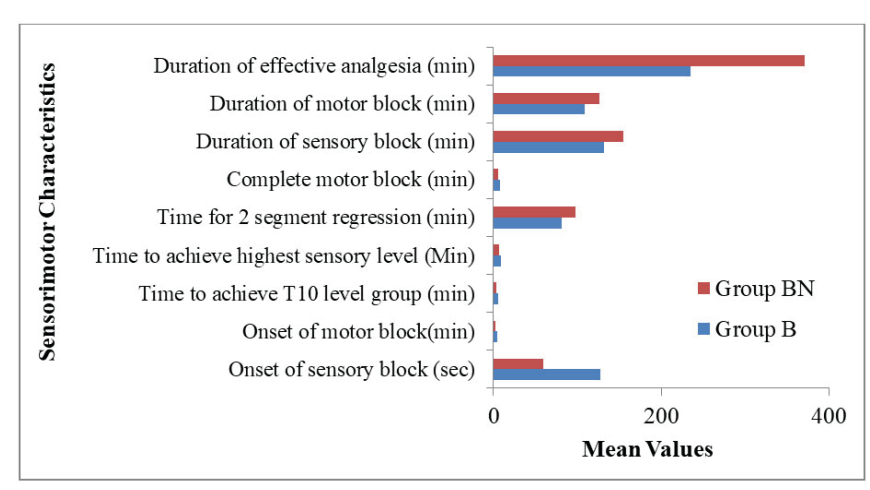

Figure 2: Comparison of sensorimotor characteristics between two groups.

The highest sensory level was T4 in the maximum number of patients in group $\mathrm{BN}(56 \%)$ while $58 \%$ of patients in group B achieved the T6 level, which was achieved by $36 \%$ patients in group BN as shown in table 1.

Table 1: Highest Sensory Level (HSL) Achieved (Dermatome)

\begin{tabular}{|l|c|c|}
\hline HSL & Group B & Group BN \\
\hline $\mathrm{T}_{4}$ & $9(18 \%)$ & $28(56 \%)$ \\
\hline $\mathrm{T}_{5}$ & $\mathrm{o}(\mathrm{o} \%)$ & $\mathrm{o}(\mathrm{o} \%)$ \\
\hline $\mathrm{T} 6$ & $29(58 \%)$ & $18(36 \%)$ \\
\hline $\mathrm{T}_{7}$ & $12(24 \%)$ & $4(8 \%)$ \\
\hline
\end{tabular}

There was a significantly higher mean VAS score observed in group B than group BN at 90 - 330 minutes. At 330 min a mean of VAS= 4 was noted in group $B$, hence rescue analgesia was given and no further evaluation for this group was done. The analysis showed that group BN had a significantly lower mean VAS score between the time intervals of $90-240$ mins, the mean VAS score of 4 was achieved at 450 minutes in group BN (Figure 3).

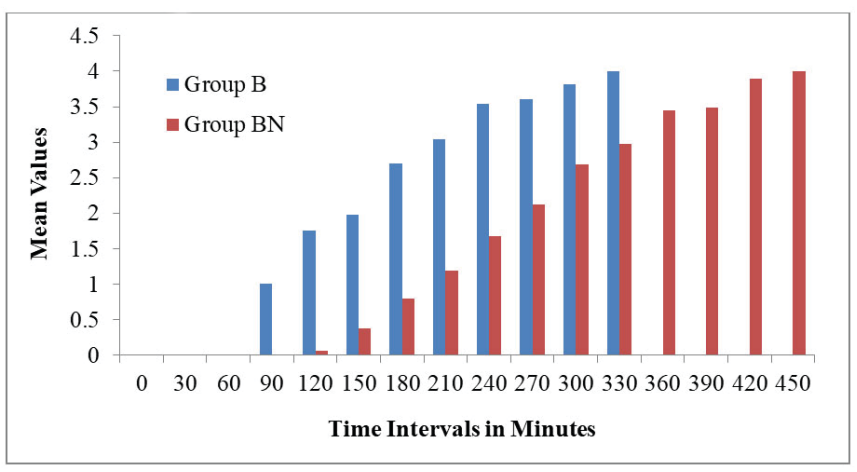

Figure 3: Mean VAS Score at Various Time Intervals.

Sedation score, hemodynamic parameters, and respiratory rate changes were comparable in two groups. In group B, 2 (4\%) patients complained nausea/ vomiting, intraoperative hypotension was noted in $2(4 \%)$ patients and post-dural puncture headache was recorded in $1(2 \%)$ patient. In group $\mathrm{BN}$, bradycardia was observed in $3(6 \%)$ patients, (Table 2).

\begin{tabular}{llcc}
\multicolumn{3}{c}{ Table 2: Incidence of Complications } & \\
\hline Complications & & Group B & Group BN \\
\hline Intra-operative & Nausea/Vomiting & $2(4 \%)$ & o (o\%) \\
& Hypotension & $2(4 \%)$ & o (o\%) \\
& Bradycardia & o (o\%) & $3(6 \%)$ \\
& Pruritus & o (o\%) & o (o\%) \\
Post-operative & $\begin{array}{l}\text { Postdural puncture } \\
\text { headache }\end{array}$ & $\mathbf{1}(\mathbf{2} \%)$ & $\mathbf{0}(\mathbf{0} \%)$ \\
& & & \\
\hline
\end{tabular}

\section{DISCUSSION}

Neuraxial anaesthesia along with neuraxial opioids is one of the important components of enhanced recovery after surgery. It is also one of the important modalities in multimodal analgesia ${ }^{10}$. With the advancement in the medical field, there has been a paradigm change from the use of general anesthesia to regional anesthesia wherever possible. The major drawback of spinal anaesthesia is hypotension and bradycardia. To some extent, it can be managed by reducing the dose of local anaesthetic. Intrathecal opioids added to local anesthetics will decrease their dose $\mathrm{e}^{11-13}$.

Nalbuphine, an agonist-antagonist drug, binds to both receptors i.e. $\mu$ and kappa, but it has a divergent action. At $\mu$ receptor, it only displaces other $\mu$ agonists from the receptor in a competitive manner without displaying any agonist activity comparable to naloxone but it has agonist activating effect when it binds to a kappa receptor. Such binding patterns and effects characterize nalbuphine as a mixed agonist-antagonist. After intrathecal administration, it binds to the kappa receptors in the brain and spinal cord. These receptors are involved in nociception, thus producing analgesia and sedation without $\mu$ side effects ${ }^{9}$ Also, it improves the quality of block, gives prolonged postoperative analgesia. It is also a cost-effective drug ${ }^{14}$. Mukherjee et al. ${ }^{15}$ used different doses of nalbuphine intrathecally $(200,400$, and $800 \mu \mathrm{g}$ ) along with $0.5 \%$ hyperbaric bupivacaine. They concluded that the duration of sensory block and the duration of effective analgesia were prolonged with $400-\mu \mathrm{g}$ and $800-\mu \mathrm{g}$ doses. They also noted that the side effects were higher with $800-\mu \mathrm{g}$ dose. Therefore, we chose to add $500 \mu \mathrm{g}$ of preservative-free nalbuphine, which is very close to the best-recognized dose earlier and is practically easier to calculate. The same dose $(0.5$ $\mathrm{mg}$ ) of intrathecal nalbuphine was used in the study done by Saad et al. ${ }^{1}$ and Dubey and Bisht ${ }^{16}$.

In our study, the onset of sensory and motor block was faster as compared to plain bupivacaine. Time to achieve T10 level, highest sensory level as well as time for the complete motor 
block was shorter in group BN as compared to group B. The differencefound to be statistically significant. Similar results are reported by Parveen et al. ${ }^{6}$ and Kumaresan et al. ${ }^{17}$. The two-segment regression of sensory block, duration of sensory and motor block was prolonged when nalbuphine was used as an adjuvant to intrathecal bupivacaine. There was a significant prolongation of effective analgesia duration too. These results correlated well with the previous studies [9, 18-20]. The study conducted by $\operatorname{Lin}^{19}$ found that nalbuphine $0.4 \mathrm{mg}$ with intrathecal tetracaine improved the quality of intraoperative analgesia. Culebras et al. ${ }^{21}$ also found that intrathecal nalbuphine $0.8-1.6 \mathrm{mg}$ improved the quality of intraoperative analgesia during cesarean deliveries with good visceral analgesia. In the present study, quality of analgesia during the procedure was excellent in $94 \%$ with nalbuphine as an adjuvant to spinal bupivacaine.

Patients receiving bupivacaine alone had significantly higher pain scores earlier than patients who received nalbuphinebupivacaine combinations as assessed by VAS. Studies done by Tiwari et al. ${ }^{18}$ and Mostafa et al. ${ }^{20}$ also reported that nalbuphine prolonged duration of analgesia with reduced VAS pain score. Sedation score, hemodynamic parameters, and respiratory rate changes were comparable between the two groups and found no significant difference among groups.

A large number of animal studies have been carried out to prove that intrathecal nalbuphine is not neurotoxic. Rawal et al. ${ }^{22}$ studied the behavioral and histopathological effects following intrathecal administration of butorphanol, fentanyl, and nalbuphine in sheep. They found that nalbuphine was the least irritating to neural tissues even at higher doses of 15-24 $\mathrm{mg}$. It was not associated with any histopathological changes in the spinal cord. Later human studies were also conducted to test the efficacy of nalbuphine which confirmed the same results. There have been no reports on the neurotoxic effects of using intrathecal nalbuphine for over 10 years. There are some studies on pregnant women, which do not reveal any unwanted action ${ }^{23}$.

\section{CONCLUSION}

With $500 \mu \mathrm{g}$ of nalbuphine added to heavy hyperbaric spinal bupivacaine $0.5 \%$ as an adjuvant, both sensory and motor block onset are faster. Time to two-segment regression and time duration of sensory and motor block are prolonged. Intraoperative analgesia is excellent with a longer duration of extended duration of postoperative analgesia. Thus, the drug nalbuphine proves to be an effective adjuvant to hyperbaric $0.5 \%$ bupivacaine for spinal anaesthesia.

\section{Limitations of the study}

There is a need for more work on the side effect profile by increasing the number of cases. In existing study rescue analgesia was the endpoint; we need to follow further to find out the total doses of analgesics needed to keep VAS score below 4. Further study is needed to determine how much we can reduce the dose of local anesthetic.

Acknowledgements: The authors would like thanks the Department of Anesthesiology, surgery, other staff of operation theatre and administration of Indira Gandhi Government Medical College and Hospital, Nagpur, Maharashtra, for permission to study and providing facility to carry out the work.

\section{Funding: No}

\section{Conflict of interest: None}

Ethical approval: Institutional ethics committee

\section{REFERENCES}

1. Saad S, Afifi E et al. Intrathecal nalbuphine as an adjuvant to bupivacaine in spinal anesthesia for lower-limb surgeries: intraoperative and postoperative effects. Benha Med J 2018; 35: 297-306.

2. Rudra A, Halder R et al. Efficacy of low dose propofol for control of emetic episodes during cesarean delivery with spinal anesthesia. Indian J Anesth. 2004; 48: 31-4.

3. Bogra J, Arora N, Srivastava P et al. Synergistic effect of intrathecal fentanyl and bupivacaine in spinal anesthesia for caesarean section. BMC Anesthesiol. 2005; 5:5.

4. Palan, A., and N.K. Agrawal. "Control of Intraoperative Shivering under Spinal Anaesthesia- A Prospective Randomized Comparative Study of Butorphanol with Tramadol." Journal of Krishna Institute of Medical Sciences University 2017; 6(1): 57-65.

5. Varghese, L, and K. Taksande. "A Comparison between Intrathecal Dexmedetomidine with Hyperbaric Bupivacaine and Intrathecal Fentanyl with Hyperbaric Bupivacaine in Lower Abdominal Surgeries: A Prospective Double-Blinded Study." Journal of Datta Meghe Institute of Medical Sciences University 2017; 12(2): 99-109.

6. Parveen S, Prasad PK, Lakshmi BS. Evaluation of the Effect of Intrathecal Nalbuphine as an Adjuvant to Spinal Bupivacaine for Post-operative Analgesia in Patients Undergoing Abdominal Hysterectomy: A Randomized, Double-Blinded Control Trial. Int J Sci Stud 2015; 3(8):141-146.

7. Rajan, R., Gosavi S et al. A Comparative Study of Equipotent Doses of Intrathecal Clonidine and Dexmedetomidine on Characteristics of Bupivacaine Spinal Anesthesia.” Journal of Datta Meghe Institute of Medical Sciences University 2018; 13(1): 4-8.

8. Swain A, Nag D et al. Adjuvants to local anesthetics: Current understanding and future trends. World J Clin Cases. 2017; 5(8): 307-323.

9. Jyothi B, Gowda S, Shaikh S. A comparison of analgesic effect of different doses of intrathecal nalbuphine hydrochloride with bupivacaine and bupivacaine alone for lower abdominal and orthopedic surgeries. Indian J Pain 2014; 28: 18-23.

10. Ituk U, Habib AS. Enhanced recovery after cesarean delivery. F1000 Faculty Rev-513. Published 2018 Apr 27.

11. Gupta K, Gupta A et al. Efficiency of nalbuphine as an adjuvant to bupivancaine in lower limb orthopaedic surgery- a prospective study. Int J Res Med Sci 2017; 5: 623-6.

12. Tang J, Weinberg L. A Literature Review of Intrathecal Morphine Analgesia in Patients Undergoing Major Open Hepato- 
Pancreatic-Biliary (HPB) Surgery, Anesth Pain Med. Online ahead of Print ; 9(6): e94441.

13. Merchea A, Lovely $\mathrm{J}$ et al. Efficacy and Outcomes of Intrathecal Analgesia as Part of an Enhanced Recovery Pathway in Colon and Rectal Surgical Patients.Surgery Research and Practice2018; 1-5.

14. Sapate M, Sahu P et al. A randomized, double blind, control study of the effects of adding nalbuphine to spinal bupivacaine for lower abdominal surgeries in elderly patients. Anaesth Pain Intensive care 2013; 17:145-148.

15. Mukherjee A, Pal A et al. Intrathecal nalbuphine as an adjuvant to subarachnoid block: what is the most effective dose? Anesth Essays Res 2011; 5:171-175.

16. Dubey R, Bisht S. The effect of addition of nalbuphine to intrathecal bupivacaine used to elderly patient in lower abdominal surgeries under spinal anesthesia. J Evol Med Dent Sci 2014; 70:14963-14970.

17. Kumaresan S, Raj A. Intrathecal Nalbuphine as an Adjuvant to Spinal Anaesthesia: What is Most Optimum Dose?. Int J Sci Stud 2017;5(1):57-60.
18. Tiwari A, Tomar G, Agrawal J. Intrathecal bupivacaine in comparison with a combination of nalbuphine and bupivacaine for subarachnoid block: A randomized prospective double blind clinical study. Am J Ther 2013; 20:592-5.

19. Lin ML. The analgesic effect of subarachnoid administration of tetracaine combined with low dose of morphine or nalbuphine for spinal anaesthesia. Ma Tsui Hsueh Tsa Chi 1992; 30:101-5.

20. Mostafa M, Mohamad M, Farrag W. Which has greater analgesic effect; Intrathecal nalbuphine or intrathecal tramadol. J Am Sci 2011;7: 480-4.

21. Culebras X, Gaggero $G$ et al. Advantages of intrathecal nalbuphine compared with intrathecal morphine, after cesarean delivery: An evaluation of postoperative analgesia and adverse effects. Anesth Analg 2000; 91: 601-5.

22. Rawal N, Nuutinen L, Raj PP. Behavioral and histopathologic effects following intrathecal administration of butorphanol, sufentanil, and nalbuphine in sheep. Anesthesiology 1991; 75:1025-1034.

23. Shakooh S, Bhole P. Intrathecal nalbuphine an effective adjuvant for post-operative analgesia. Innov J Med Health Sci 2014; 4: 79-82. 